\title{
DERIVATIONS ON COMMUTATIVE BANACH ALGEBRAS
}

\section{A. KHOSRAVI}

ABSTRACT. Let $A$ be a commutative Banach algebra with radical $R$ and $D$ be a derivation on $A$. If $K=\left\{x \in R\right.$ : for every $\left.n>1, D^{n} x \in R\right\}$, then $D A \subseteq R$ if and only if $K$ is closed.

1. Introduction. In [10] Singer and Wermer showed that the range of a continuous derivation on a commutative Banach algebra is contained in the radical. They conjectured that the assumption of continuity is unnecessary.

In [5] Johnson proved the following result.

TheOREM. If $A$ is a commutative Banach algebra with identity $e$ and if $D: A \rightarrow A$ is a derivation, then there exist orthogonal idempotents $e_{0} \ldots e_{m}$ in $A$ with sum e such that $D\left(e_{0} A\right)$ is contained in the radical of $e_{0} A$ and such that each algebra $e_{i} A \ldots e_{m} A$ has just one maximal ideal. If $A$ is semisimple, then $D$ is continuous.

Let $\mathbf{C}$ be the complex field and $\mathbf{N}$ the set of all nonnegative integers.

Throughout this paper we suppose that $A$ is a commutative Banach algebras. $R$ and $\Delta A$ will denote, respectively, the radical and the spectrum of $A$.

If $S$ is a linear operator from a Banach space $X$ into a Banach space $Y$, then the separating space $\subseteq(S)$ of $S$ is defined by

$$
\Im(S)=\left\{y \in Y: \text { there are } x_{n} \rightarrow 0 \text { in } X \text { with } S x_{n} \rightarrow y \text { in } Y\right\} \text {. }
$$

It is a great pleasure to thank Dr. G. R. Allan who recommended the problem to me and helped me with his comments and encouragement during the process of this work at Cambridge University.

\section{Main results.}

Lemma 1. Let $D: A \rightarrow A$ be a derivation and let

$$
K=\left\{x \in R: \text { for every } n \geqslant 1, D^{n} x \in R\right\} .
$$

Then $K$ is a prime ideal of $A$.

Proof. For convenience take $D^{0} x=x(x \in A)$. It is plain that $K$ is an ideal of $A$. Let $a_{1} a_{2} \in K$ for some $a_{1}, a_{2} \in A$ and let $a_{1} \notin K$. We must show that $a_{2} \in K$. Since $a_{1} \notin K$ there is an $n \geqslant 0$ such that, for each $m<n, D^{m} a_{1} \in R$ but $D^{n} a_{1} \notin$ $R$. Now by induction on $i$ we prove that, for each $i>0, D^{i} a_{2} \in R$.

Received by the editors January 22, 1980 and, in revised form, February 25, 1981. 1980 Mathematics Subject Classification. Primary 46J05, 46G05. 
For $i=0:$ Since

$$
D^{n}\left(a_{1} a_{2}\right)=a_{2}\left(D^{n} a_{1}\right)+\sum_{j=1}^{n}\left(\begin{array}{l}
n \\
j
\end{array}\right) D^{j}\left(a_{2}\right) D^{n-j} a_{1}
$$

and $a_{1} a_{2} \in K$, then $a_{2} D^{n} a_{1} \in R$.

On the other hand $R$ is a primitive ideal so $R$ is a prime ideal. Therefore by the fact that $D^{n} a_{1} \notin R$ we have $a_{2} \in R$.

Let $D^{0} a_{2} \cdots D^{i-1} a_{2} \in R$. We proceed for $i$. By Leibnitz's formula

$$
\begin{aligned}
D^{n+1}\left(a_{1} a_{2}\right)= & \sum_{j=0}^{i-1}\left(\begin{array}{c}
n+i \\
j
\end{array}\right) D^{j}\left(a_{2}\right) D^{n+i-j}\left(a_{1}\right)+\left(\begin{array}{c}
n+i \\
i
\end{array}\right) D^{i}\left(a_{2}\right) D^{n}\left(a_{1}\right) \\
& +\sum_{j=i+1}^{n+i}\left(\begin{array}{c}
n+i \\
j
\end{array}\right) D^{j}\left(a_{2}\right) D^{n-j+i}\left(a_{1}\right) .
\end{aligned}
$$

Since $a_{1} a_{2} \in K$, then $D^{n+i}\left(a_{1} a_{2}\right) \in R$. Now by the assumption and induction hypothesis we get $\left(\begin{array}{c}n+i \\ i\end{array}\right) D^{i}\left(a_{2}\right) D^{n} a_{1} \in R$. As before this implies that $D^{i}\left(a_{2}\right) \in R$. Hence we have the result.

LEMMA 2. Let $A$ have a unique maximal ideal which is its radical $R$. If $D$ is a derivation on $A, K=\left\{x \in R\right.$ : for every $\left.n \geqslant 1, D^{n} x \in R\right\}$ and $\subseteq(D) \cap R \subseteq K$, then $D A \subseteq R$.

Proof. Suppose that $\subseteq(D) \cap R \subseteq K$. Then $\subseteq(D)$ is an ideal in $A$ so $\subseteq(D)=A$ or $\subseteq(D) \subseteq R$ because $R$ is the unique maximal ideal of $A$. In the first case $R=\subseteq(D) \cap R \subseteq K \subseteq R$ so $K=R$. In the second case $\subseteq(D)=\subseteq(D) \cap R \subseteq K$.

The operator $D_{1}$ from $A$ into $A / \bar{K}$ defined by $D_{1}(a)=D(a)+\bar{K}(a \in A)$, where $\bar{K}$ denotes the closure of $K$, is continuous, by [9, Lemma 1.4]. Since $D(K) \subseteq K$, it follows that $D_{1}(K)=0$ so $D_{1}(\bar{K})=0$.

Thus $D$ defines a continuous derivation $D_{0}$ from $A / \bar{K}$ into $A / \bar{K}$ by

$$
D_{0}(x+\bar{K})=D x+\bar{K} \text { for all } x \in A \text {. }
$$

Singer and Wermer's Theorem [2, Theorem 18.16] implies that $D_{0}(A / \bar{K}) \subseteq R / \bar{K}$ because $R / \bar{K}$ is clearly the unique maximal ideal of $A / \bar{K}$. Since $\bar{K} \subseteq R$, then $D A \subseteq R$.

TheOREM 1. If $D$ is a derivation on $A$ and $K=\{x \in R$ : for every $m>1$, $\left.D^{m} x \in R\right\}$, then $D A \subseteq R$ if and only if $\subseteq(D) \cap R \subseteq K$.

Proof. Since a derivation on a Banach algebra maps its identity to zero then we can, and will, suppose that $A$ has an identity, say 1.

If $D A \subseteq R$, then $K=R$ so $\subseteq(D) \cap R \subseteq R=K$.

Conversely suppose that $\subseteq(D) \cap R \subseteq K$. By Johnson's Theorem there exist orthogonal idempotents $e_{0}, e_{1}, \ldots, e_{m}$ in $A$ with sum 1 such that $D\left(e_{0} A\right)$ is contained in the radical of $e_{0} A$ and such that each algebra $e_{1} A, \ldots, e_{m} A$ has a unique maximal ideal.

Let $1 \leqslant i \leqslant m$ and take $K_{i}=\left\{x \in \operatorname{Rad}\left(A e_{i}\right)\right.$ : for all $\left.n, D^{n} x \in \operatorname{Rad}\left(e_{i} A\right)\right\}$. Hence $K_{i}=e_{i} K$ and so $\subseteq(D) \cap \operatorname{Rad}\left(e_{i} A\right) \subseteq \operatorname{Rad}\left(e_{i} A\right)$. Therefore by the above lemma $D\left(e_{i} A\right) \subseteq \operatorname{Rad}\left(e_{i} A\right)$. Since $i$ was arbitrary, then $D A \subseteq R$. 
The following definition is due to Cusack [3].

Definition. A closed ideal $I$ of a Banach algebra $B$ is called a separating ideal if, for every sequence $\left(a_{n}\right)$ in $B$, there is an $N>1$ such that $\left(I a_{1}, \ldots, a_{n}\right)^{-}=$ $\left(I a_{1} \ldots a_{N}\right)^{-}(n \geqslant N)$.

Let $R$ be the ideal spanned by $n$-fold products of elements of $R$.

LEMMA 3. If $\cap_{n>1} R^{n}=\{0\}$ and $D$ is a derivation on $A$, then $S(D)$ is nilpotent and $D A \subseteq R$.

Proof. Since $S(D) \cap R \subseteq R, S(D) \cap R$ is a radical commutative Banach algebra. Let $x \in \mathfrak{S}(D) \cap R$. Since $S(D)$ is a separating ideal by [6, Lemma 1], there is a positive integer $N$ such that

$$
\left(G(D) x^{N}\right)^{-}=\left(G(D) x^{n}\right)^{-} \text {for all } n>N .
$$

Hence by the Mittag-Leffler Theorem [4, Theorem 5.3]

$$
\left(\Im(D) x^{N}\right)^{-}=\bigcap_{n>1}\left(\Im(D) x^{n}\right)^{-}=\bigcap_{n>1}\left(\Im(D) x^{n}\right)^{-}=\{0\} .
$$

Therefore $x^{N+1}=0$ and $x$ is nilpotent. Since $x$ was arbitrary $\subseteq(D) \cap R$ is a nilpotent ideal. Since $K$ is a prime ideal of $A$, then $\subseteq(D) \cap R \subseteq K$.

Now by Theorem 1 this implies that $D A \subseteq R$ and then $\subseteq(D) \cap R=\subseteq(D)$ since S(D) $\subseteq \overline{D A} \subseteq R$.

COROLlaRY 1. If $I=\bigcap_{n>1} R^{n}$ is closed, then for every derivation $D$ on $A$, $D A \subseteq R$.

Proof. Since for all $n, R^{n}$ is an ideal of $A$ then $I$ is an ideal of $A$. If $D$ is a derivation on $A$ then $D I \subseteq I$ because $D\left(R^{n}\right) \subseteq R^{n-1}$ for $n>2$.

Thus $D$ gives a derivation $D_{1}$ on $A / I$ defined by

$$
D_{1}(x+I)=D x+I \quad(x \in A) .
$$

It is clear that $\operatorname{Rad}(A / I)=R / I$.

Now we prove that $\bigcap_{n>1}(R / I)^{n}=\{0\}$. Let $\xi \in \cap_{n>1}(R / I)^{n}$; then for each $n$, there is an element $\alpha_{n} \in R^{n}$ such that $\xi=\alpha_{n}+I$. Thus $\alpha_{1}-\alpha_{n} \in I$ for all $n$, $I \subseteq R^{n}$, so $\alpha_{1} \in R^{n}$ for all $n$, i.e. $\alpha_{1} \in I$ and so $\xi=0$.

Then, by Lemma $3, D_{1}(A / I) \subseteq R / I$ which implies $D A \subseteq R$, and we have the result.

In [7] Loy proved that every derivation on a Banach algebra of formal power series is continuous. Since for a Banach algebra of formal power series $\bigcap_{n>1} R^{n}=$ $\{0\}$ and $R$ is an integral domain, the following result is a generalization of his result.

Corollary 2. If $\cap_{n>1} R^{n}=\{0\}$ and $R$ is an integral domain, then every derivation $D$ on $A$ is continuous.

Proof. If $D$ is a derivation on $A$, then $S(D)$ is nilpotent. On the other hand $R$ is an integral domein, so $\subseteq(D)=\{0\}$ and $D$ is continuous. 
TheOREM 2. If $D$ is $a$ derivation on $A$ and $K=\{x \in R$ : for every $n>1$, $\left.D^{n} x \in R\right\}$, then $D A \subseteq R$ if and only if $K$ is closed.

Proof. If $D A \subseteq R$, then $K=R$ is closed.

Conversely if $K$ is closed, then since $D K \subseteq K, D$ gives a derivation $D_{1}$ on $A / K$ defined by $D_{1}(x+K)=D x+K(x \in A)$.

Since $K \subseteq R$, then $\operatorname{Rad}(A / K)=R / K$.

We prove $\cap_{n>1}(R / K)^{n}=\{0\}$. Let $x \in \cap_{n>1}(R / K)^{n}$. Then there are elements $\alpha_{n} \in R^{n}$ such that $x=\alpha_{n}+K$. Now for every $n>1, \alpha_{1}-\alpha_{n+1} \in K$; therefore $D^{n}\left(\alpha_{1}-\alpha_{n+1}\right) \in K$.

On the other hand $D^{n} \alpha_{n+1} \in R$ so $D^{n} \alpha_{1} \in R$. Since $n$ was arbitrary, then $\alpha_{1} \in K$ and $x=0$. But $x$ was arbitrary, so $\cap_{n>1}(R / K)^{n}=\{0\}$, and by Lemma 3 , $D A+K \subseteq R$. Then $D A \subseteq R$ as we wanted.

Corollary. Let every prime ideal of $A$ be closed. Then for every derivation $D$ on $A, D A \subseteq R$.

Proof. Take $K=\left\{x \in R\right.$ : for every $\left.n>1, D^{n} x \in R\right\}$. Since $K$ is a prime ideal of $A$, then $K$ is closed and, by Theorem 2, we have the result.

EXAmple. Let $l^{2}$ be the well-known Hilbert space. Define $T: l^{2} \rightarrow l^{2}$ by

$$
T \mathbf{x}=\left(0, \alpha_{1} x_{1}, \alpha_{2} x_{2}, \ldots\right) \text { for } \mathbf{x}=\left(x_{1}, x_{2}, \ldots\right) \text {, }
$$

where $\left(\alpha_{n}\right)$ are nonzero elements of $\mathbf{C}$ such that $\alpha_{n} \rightarrow 0$.

Then $T$ is quasinilpotent and $\cap_{n>1} T^{n}\left(l^{2}\right)=(0)$. Let $B$ be the norm-closed subalgebra of $L\left(l^{2}\right)$ generated by $\{I, T\}$ where $I: l^{2} \rightarrow l^{2}$ is the identity map. Now we prove that $\operatorname{Rad} B=\overline{B T}$.

Since $T$ is quasinilpotent, then $\overline{B T} \subseteq \operatorname{Rad} B$.

On the other hand, let $x \in \operatorname{Rad} B$, so there are polynomials $P_{n}(T)$ such that $P_{n}(T) \rightarrow x$. We can write $P_{n}(T)=B_{n} I+q_{n}(T)$, where $q_{n}(T) \subseteq B T$.

Let $\varphi$ be a multiplicative linear functional on $B$, so $\varphi\left(p_{n}(T)\right)=B_{n} \rightarrow \varphi(x)=0$. Therefore $q_{n}(T) \rightarrow x$. But $q_{n}(T) \subseteq B T$ and so $x \subseteq \overline{B T}$. Moreover $\cap_{n>1}(\operatorname{Rad} B)^{n}=$ $\{0\}$ and $B=C \cdot I \oplus \operatorname{Rad} B$. Hence for every derivation $D$ on $B$, by Lemma 3 , $D B \subseteq \operatorname{Rad} B$. From Theorem 1, we get the following result.

TheOREM $1^{\prime}$. If $D$ is a derivation on $A$, then $D A \subseteq R$ if and only if for every $n>1$ and $\varphi \in \Delta A, \varphi \circ D^{n}$ is continuous.

Proof. If $D A \subseteq R$, then for every $n>1$ and $\varphi \in \Delta A, \varphi \circ D^{n}=0$ which is continuous.

Conversely, for all $n \geqslant 1$ and $\varphi \in \Delta A$, let $\varphi \circ D^{n}$ be continuous. Let $x \in S(D)$ $\cap R, n>1$, and $\varphi \in \Delta A$. By the fact that $\varphi \circ D^{n}$ and $\varphi \circ D^{n+1}$ are continuous, we have $\varphi \circ D^{n}(x)=0$. Since $\varphi$ and $n$ were arbitrary and $x \in R$, we conclude that $x \in K$ and by Theorem 1 we have the result.

For generalizing Singer and Wermer's Theorem first we prove the following lemma.

Lemma 4. Let $A$ have a unique maximal ideal which is its radical. If $D$ is a derivation on $A$ and, for some $n, D^{n}$ is continuous, then $D A \subseteq R$. 
Proof. Let $\Delta A=\{\varphi\}$. Since $D^{n}$ is continuous, for each $a \in A$ and $m \in \mathbf{N}$

$$
\left\|D^{m}(a)\right\| \leqslant \max _{0<j<n}\left\|D^{j}(a)\right\| \cdot\left\|D^{n}\right\|^{i}
$$

where $m=i n+r, i \in \mathbf{N}, 0 \leqslant r<n$. So $\exp D$ is well defined and, for every $z \in \mathbf{C}$, the map $a \mapsto(\exp (z D) a)(a \in A)$ is a multiplicative linear functional. Therefore

$$
\varphi(\exp (z D) a)=\varphi(a) \quad(a \in A) .
$$

Since $\varphi(a)$ is independent of $z$, this gives $\varphi \circ D=0$, i.e. $D A \subseteq R$.

THEOREM 3. If $D$ is a derivation on $A$ and, for some $n, D^{n}$ is continuous, then $D A \subseteq R$.

Proof. As we state in the proof of Theorem 1 we can, and will, suppose that $A$ has an identity, say 1. By Johnson's Theorem, there exist orthogonal idempotents $e_{0}, e_{1}, \ldots, e_{m}$ in $A$ with sum 1 such that $D\left(e,{ }_{0} A\right)$ is contained in the radical of $e_{0} A$ and such that each algebra $e_{1} A, \ldots, e_{m} A$ has a unique maximal ideal. Let $1 \leqslant i \leqslant m$. Since $D^{n}$ is continuous, the restriction of $D^{n}$ to $e_{i} A$ is continuous and by the above lemma $D\left(e_{i} A\right) \subseteq \operatorname{Rad}\left(e_{i} A\right)$ since $i$ was arbitrary, then $D A \subseteq R$.

\section{REFERENCES}

1. G. R. Allan, Embedding the algebra of formal power series in a Banach algebra, Proc. London Math. Soc. 25 (1972), 329-340.

2. F. F. Bonsall and J. Duncan, Complete normed algebras, Springer-Verlag, New York, 1973.

3. J. Cusack, Automatic continuity and topologically simple radical Banach algebras, J. London Math. Soc. (2) 16 (1977), 493-500.

4. H. G. Dales, Automatic continuity: A survey, Bull. London Math. Soc. 10 (1978), 129-183.

5. B. E. Johnson, Continuity of derivations on commutative Banach algebras, Amer. J. Math. 91 (1969), $1-10$.

6. N. P. Jewell and A. M. Sinclair, Epimorphisms and derivations on $L^{1}(0,1)$ are continuous, Bull. London Math. Soc. 8 (1976), 135-139.

7. R. J. Loy, Continuity of derivations on topological algebras of power series, Bull, Austral. Math. Soc. 1 (1969), 419-424.

8. A. M. Sinclair, Continuous derivatives on Banach algebras, Proc. Amer. Math. Soc. 20 (1969), $166-170$.

9. __ Automatic continuity of linear operators, London Math. Soc. Lecture Note Series, vol. 21, Cambridge Univ. Press, London, 1976.

10. M. Singer and J. Wermer, Derivatives on commutative normed algebras, Math. Ann. 129 (1955), 260-264.

Department of Pure Mathematics and Mathematical Statistics, University of Cambridge, Cambridge CB2 1SB, England

Current address: Deparment of Mathematics, University for Teacher Education, 49 Mobarezan Avenue, Tehran, Iran 